\title{
Spatial and physical frames of reference in positioning a limb
}

\author{
STEVEN R. GARRETT \\ University of Connecticut, Storrs, Connecticut \\ C. PAGANO \\ Clemson University, Clemson, South Carolina \\ and \\ GARY AUSTIN and M. T. TURVEY \\ University of Connecticut, Storrs, Connecticut
}

\begin{abstract}
Splints attached to the right forearm were used to rotate the forearm's physical reference frame, as defined by the eigenvectors of its inertia tensor, relative to its spatial reference frame. In two experiments, when subjects were required to orient the forearm parallel to, or at $45^{\circ}$ to, the environmental horizontal, they produced limb orientations that were systematically deflected from the forearm's longitudinal spatial axis in the direction of the forearm's physical axes. The position sense seems to be based on inertial eigenvectors rather than on joint angles or gravitational torques.
\end{abstract}

How is it that one can position a limb in space relative to the body or the environment without vision? When a person holds and moves a cup of coffee outside of the field of view, the shoulder, elbow, and wrist function synergistically to keep the wrist and cup oriented parallel to the ground. This nonvisual ability to achieve and maintain orientations of the limbs that have directional significance for a variety of tasks is not exhibited by people with peripheral neuropathy who lack muscular proprioception and cutaneous touch while still possessing all the means for bringing about muscular contractions. For them, directing the limbs relative to the body and to adjacent objects is possible only through continuous visual control and then only in a most restricted sense (Bernstein, 1996; Cole, 1995; Cole \& Paillard, 1995). The control of the limbs by visual perception is a poor substitute for the control of the limbs by haptic perception.

In the contemporary view, the nonvisible perception of a limb segment's position is tied to the patterned distribution of afferent activity over the muscles that control movement at the joint (see, e.g., Burgess, Clark, Simon, \& Wei, 1982; Feldman \& Latash, 1982a, 1982b; Gilhodes, Coiton, Roll, \& Ans, 1992). At issue, theoretically, is what this activity specifies. It does not seem to specify joint angle. Research has shown that the perception of relative forearm orientation (the angle that it makes to the upper

\footnotetext{
This research was supported by NSF Grant SBR 97-09678. We wish to acknowledge the conceptual and artistic contributions of Claudia Carello and to thank the anonymous reviewers for their helpful comments. Correspondence should be addressed to S. R. Garrett, Center for the Ecological Study of Perception and Action, U-20, University of Connecticut, 406 Babbidge Rd., Storrs, CT 06268 (e-mail: srg93001 @) uconnvm.uconn.edu).
}

arm) is less accurate than the perception of absolute forearm orientation (the angle that it makes with the vertical or the trunk) (Soechting, 1982) and that the errors in matching elbow joint angles are strongly biased in the direction of matching forearm inclinations (Worringham \& Stelmach, 1985; Worringham, Stelmach, \& Martin, 1987). Perhaps the patterning of afferent activity over the muscles of a joint is specific to the muscular and external forces acting on the limb segment. Although these forces most certainly affect limb control in terms of motoneuron recruitment levels, they are ambiguous with respect to limb position (see, e.g., Feldman \& Levin, 1995). A related possibility is that the specificity is in terms of the gravitational torque acting on the limb segment (Worringham et al., 1987). Gravitational torque, however, is equivocal with respect to limb position. For example, it has the same value for different elbow angles in planes parallel to the gravitational vertical and does not distinguish among elbow angles in planes of motion perpendicular to the gravitational vertical (e.g., Feldman \& Levin, 1995; Pagano \& Turvey, 1995). It is also of seemingly little use in zeroand micro-gravity situations.

Recent evidence has shown that the perceived orientation of a limb can be influenced systematically by an attached splint that essentially alters the distribution of the limb's mass (Pagano, Garrett, \& Turvey, 1996; Pagano \& Turvey, 1995). Each limb segment can be represented by an inertial ellipsoid about the segment's point of rotation. The ellipsoid captures the segment's mean mass distribution, which is normally centered on its longitudinal axis. The inertial ellipsoid's axes are the principal directions or eigenvectors $e_{k}(k=1,2,3)$ of the inertia tensor defined at that rotation point, with each axis equal in length to twice the inverse of the square root of 
the axis's corresponding eigenvalue. Pagano and Turvey (1995) investigated the perceptual consequences of altering the mass distribution of an occluded arm for positioning tasks with visual targets. They showed that breaking the normal relation between the right arm's inertia ellipsoid and its spatial axes affected the positioning of the arm relative to the visual targets systematically. Specifically, Pagano and Turvey rotated the arm's physical frame $\left\{e_{k}\right\}$ relative to its spatial frame $\left\{\sigma_{k}\right\}$ (defined by the arm's longitudinal axis and the spatial axes orthogonal to it) and observed that the direction of the pointing error followed $\left\{e_{k}\right\}$. The fact that individuals tended to align the arm's inertia ellipsoid rather than its long spatial axis with the visible target means that their impression of the arm's position was not tied to the angle that the arm made with the shoulders. In a subsequent study, using a rhythmic variant of the forearm matching procedure, Pagano et al. (1996) showed that with $\left\{e_{k}\right\}$ manipulated through attached splints, subjects tended to match the $\left\{e_{k}\right\} \mathrm{s}$ of the forearms rather than the elbow angles. The importance of these experiments by Pagano and colleagues is threefold. First, they corroborate previous research (e.g., Soechting, 1982; Soechting \& Ross, 1984; Worringham \& Stelmach, 1985; Worringham et al., 1987) suggesting that joint angle or relative orientation is not the relevant quantity involved in the position sense. Second, they advance previous research by identifying the significance to the position sense of the inertia tensors of the segments of the body relative to their proximal joints. Third, they link perception of the directions of the body to the perception of spatial aspects of handheld, wielded objects and, thereby, to the touch subsystem referred to as dynamic (Gibson, 1966) or kinesthetic (Loomis \& Lederman, 1986). A large body of data reveals that the perception of wielded objects is constrained by the eigenvalues and eigenvectors of the objects' inertia tensors (for summaries, see Turvey, 1996; Turvey \& Carello, 1995).

The two experiments reported in the present article were directed at the nonvisual ability to orient a single limb segment in a designated plane. Specifically, subjects were instructed to orient the right forearm in a plane parallel to the sagittal plane either horizontally (i.e., parallel to the ground/perpendicular to the trunk) or at $45^{\circ}$. At issue was whether this requirement to position the forearm in a plane defined unambiguously with respect to both the environment and the seated body with upright trunk would render the position sense immune to inertial manipulations of the limb. An efferent copy (von Holst, 1954) shaped by spatial memory and by felt body position with respect to the environment might override the influence of the inertial eigenvectors. Failure to find an eigenvector effect in the present experimental setting would bring into question the status of the inertia tensor quantitation of limbs as a general factor in the perceptual control of limb position.

\section{EXPERIMENT 1}

Through cross-like splints attached to the forearms, the forearm's physical frame $\left\{e_{k}\right\}$ was rotated about the elbow $6.3^{\circ}$ clockwise $(\mathrm{CW}), 6.3^{\circ}$ counterclockwise $(\mathrm{CCW})$, and $0^{\circ}$ relative to the forearm's spatial frame $\left\{\sigma_{k}\right\}$ in the manner shown in Figure 1. (The descriptors $\mathrm{CW}$ and $\mathrm{CCW}$ are with respect to viewing the experimental arrangement from the subject's right-hand side, as depicted in Figure 1; this convention is used throughout the present article.) On the hypothesis that forearm position sense is tied to the inertia tensor, the rotation of $\left\{e_{k}\right\}$ by means of an attached splint should alter the perceived forearm orientation and, thereby, the subject's ability to match $\left\{\sigma_{k}\right\}$ with the target positions of $0^{\circ}$ and $45^{\circ}$. Specifically, the forearm should be deflected from either target position in a direction opposite to the rotation of $\left\{e_{k}\right\}$. For example, if $\left\{e_{k}\right\}$ is rotated CW (or downward), the forearm's perceived position should be rotated CW relative to $\left\{\sigma_{k}\right\}$, resulting in an actual CCW (upward) placement of $\left\{\sigma_{k}\right\}$ to a position higher than the requested $0^{\circ}$ or $45^{\circ}$. Stated differently, a lower orientation of the forearm's physical frame results in feeling the forearm at a lower orientation than it is, leading to the forearm's being placed in a higher position than it should be in order to feel the forearm in the intended orientation.

In the experiment, the two forearm directions of parallel to the ground and $45^{\circ}$ to the ground were to be achieved from two spatial positions of the elbow - that is, with two different elbow angles. If elbow angle is not the relevant variable, and if $\left\{e_{k}\right\}$ is the relevant variable, the effects of $\left\{e_{k}\right\}$ on producing the orientations of $0^{\circ}$ and $45^{\circ}$ should be independent of the two spatial positions. In sum, the expectations were that (1) the forearm would be oriented higher with $\left\{e_{k}\right\}$ rotated $6.3^{\circ} \mathrm{CW}$ (i.e., downward from the arm's longitudinal axis) than with $\left\{e_{k}\right\}$ rotated $6.3^{\circ} \mathrm{CCW}$ (i.e., upward from the arm's longitudinal axis), with the $0^{\circ}$ deflection leading to an intermediate orientation; (2) the effect of $\left\{e_{k}\right\}$ 's rotation would be the same for both intended forearm orientations; and (3) the effect of $\left\{e_{k}\right\}$ 's rotation would be the same for both elbow positions.

An important additional feature of the present task is that it permits a corresponding evaluation of the contribution of gravitational torque. The horizontal alignment of the forearm will be associated with larger magnitudes of gravitational torque than the $45^{\circ}$ alignment. Consequently, if gravitational torque matters (see, e.g., Worringham et al., 1987), the effect of the splints should depend on the intended forearm orientation; that is, given any splint--any loading of the forearm-the perceptual effect with foream horizontal should be different from that with the forearm inclined at $45^{\circ}$. The contrasting Prediction 2 above follows from the fact that, unlike gravitational torque, the orientation of the inertia ellipsoid relative to the forearm's longitudinal axis is invariant over forearm inclination. 


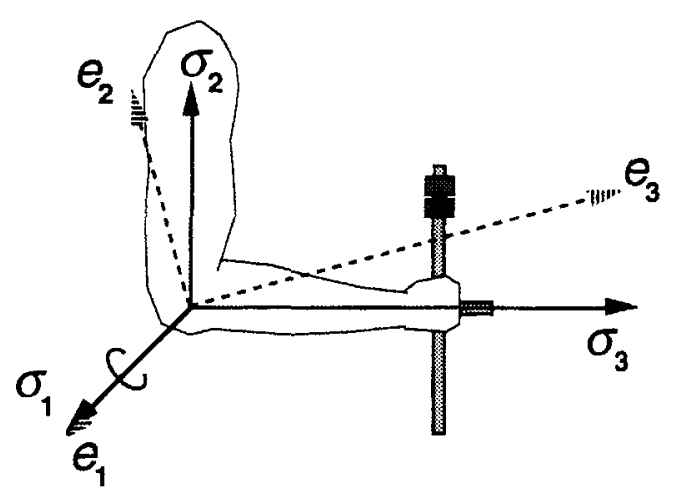

Figure 1. The physical frame $\left\{e_{k}\right\}$ rotated counterclockwise with respect to the spatial frame $\left\{\sigma_{k}\right\}$ as a result of the mass distribution of the attached splint. In both experiments, $e_{1}$ remains coincident with $\sigma_{1}$ so that the rotation of the physical reference frame is within the sagittal plane about the $\sigma_{1} / e_{1}$ axes.

\section{Method}

Subjects. Nine students ( 5 men and 4 women) at the University of Connecticut participated on a volunteer basis. All 9 subjects were right handed. No subject had any foreknowledge of the specific hypothesis in question, nor of the experimental conditions used.

Materials. The attached splints were cross-shaped objects as depicted in Figure 1 and were similar to those used in Pagano et al. (1996). Three such splints were constructed out of oak dowels $32.5 \mathrm{~cm}$ in length with a radius of $.6 \mathrm{~cm}$. The cross-piece extended $16.25 \mathrm{~cm}$ to either side of the center of a metal brace, and the stem extended $8 \mathrm{~cm}$ forward and $24.5 \mathrm{~cm}$ backward from the center of the metal brace. The total mass of each wooden splint was $120 \mathrm{~g}$. With the cross-piece aligned vertically, metal rings were attached as follows: $200 \mathrm{~g}$ above the midpoint defining Splint $\mathrm{A}$ (for above or $\mathrm{CCW}$ rotation of $\left.\left\{e_{k}\right\}\right), 100 \mathrm{~g}$ above and $100 \mathrm{~g}$ below the midpoint defining Splint S (for symmetrical or no rotation of $\left\{e_{k}\right\}$ ), or $200 \mathrm{~g}$ below the midpoint defining Splint B (for below or CW rotation of $\left\{e_{k}\right\}$ ). The metal rings were placed $15 \mathrm{~cm}$ from the midpoint of the cross. With the added metal rings, the total mass of each splint was $320 \mathrm{~g}$. On any given trial, a splint was secured to the subject's right forearm with Velcro straps so that (1) the stem extended backward against the inside of the forearm coincident with the longitudinal axis of the arm and forward from between the middle and index fingers, and (2) the cross-piece extended laterally from either side of the closed fist and was held to remain parallel to the sagittal plane.

Eigenvector calculations. The eigenvectors $e_{k}$ and rotation of $\left\{e_{k}\right\}$ were computed for each splint + forearm combination using regression equations and procedures provided by Reynolds (1978; see also Chandler, Clauser, McContville, Reynolds, \& Young, 1975; Clauser, McContville, \& Young, 1969) applied to the body dimensions of an averaged-sized person. (For an overview of the regression equations and body dimensions used in the calculations see Barac-Cikoja \& Turvey, 1993, Pagano et al., 1996, Pagano \& Turvey, 1995). From these regression equations, the mass, distances of the centers of mass $(\mathrm{CM})$ of the forearm and the hand from the point of rotation in the elbow, and principal moments of inertia for the limb segments were computed. The parallel axis theorem was then used to transform the principal moments of inertia for each segment about its respective CM to moments and products of inertia about the elbow, and these quantities were combined with those for the cross and attached metal rings to get the elements of the inertia tensor (the moments of inertia and the products of inertia) about the rotation point $O$ at the elbow for the three splint + forearm combinations. Each inertia tensor was diagonalized to arrive at its eigenvalues and eigenvectors. The eigenvectors, expressed as coordinates in $O x y z$, were transformed into angles about $O$. In each case, $e_{1}$ remained perpendicular to the longitudinal axis of the arm, and $e_{2}$ and $e_{3}$ were reoriented by the same amount within a plane perpendicular to $e_{1}$, where $0^{\circ}$ would be coincident with the longitudinal axis of the arm. From these calculations, it was estimated that the mass conditions of the three splints caused $\left\{e_{k}\right\}$ of the arm to be oriented $6.3^{\circ}$ above the arm's longitudinal axis (Splint $A$ ), $0^{\circ}$ (Splint $S$ ), or $6.3^{\circ}$ below (Splint $B$ ) the arm's longitudinal axis. Table $\perp$ presents the three eigenvalues and $e_{3}$ for each splint + forearm.

Procedure. Each subject sat in a chair with a vertical back. The subject was blindfolded. The right elbow was placed on an arm rest that could be adjusted to produce two distinct elbow positions relative to the trunk according to the requirement that the upper arm be aligned vertically (elbow backward) or at $45^{\circ}$ (elbow forward) to the vertical (see Figure 2). This manipulation defined the variable elbow position. Prior to a trial, Splint A, Splint S, or Splint B was attached to the subject's right forearm in the manner described above. At the start of a trial, the subject's right elbow was placed at one of the two positions, with the right forearm as close to vertical as possible - that is, near vertical in the elbow forward case or near maximum flexion in the elbow backward case. In the course of a trial, at different temporal points, the subject had to produce forearm orientations of $0^{\circ}$ (i.e., parallel to the ground plane or perpendicular to the trunk) and $45^{\circ}$ (defined relative to the ground plane or the trunk). This requirement defined the variable forearm orientation. The experimenter began the trial by switching on a metronome that repeatedly sounded a quadruplet of tones, with $2 \mathrm{sec}$ between tones. This continuous repetitive pattern lasted for the trial duration of $30 \mathrm{sec}$. When the subject was ready, Tone 1 acted as the signal to move the right forearm about the elbow from an upright (flexion) starting position to an orientation that the subject judged to be horizontal to the ground plane. Tone 2 signaled the subject to move the forearm back to the starting position. Tone 3 signaled the subject to move the forearm about the elbow to an orientation that he/she judged to be $45^{\circ}$ with respect to the ground plane. Tone 4 signaled the subject to again return to the flexed, upright starting position. This cycle was repeated without interruption for the duration of the trial, with 2-sec intervals between tones and cycles. Prior to the experiment proper, each subject was given practice in producing the arm motion without a splint or the blindfold. No feedback was given concerning chosen target angles. The subjects were instructed only with respect to the sequencing of the movements. On every trial in the experiment proper, there was at least one preliminary cycle of four tones. If it was completed successfully, the subject was asked to indicate whether he/she felt that the task was being done correctly; if so, the sonic digitizer was switched on by the experimenter to begin data collection. If the subject felt otherwise, a further cycle was performed and the question was asked again, and so on until the subject was confident. Typically, one cycle sufficed. The shoulder and wrist joints remained fixed by the apparatus during every trial. Between trials, subjects rested for about 2 min with longer breaks allowed as needed to avoid fatigue. The experiment lasted about

Table 1

Major $\left(I_{1}\right)$, Intermediate $\left(I_{2}\right)$, and Minor $\left(I_{3}\right)$ Eigenvalues $\left(\mathrm{g} \cdot \mathrm{cm}^{2} / 1,000\right)$ for the Three Splints Plus Forearm

Relative to the Rotation Point in the Elbow Together With the Deflections of the Forearm's Eigenvector $e_{3}$ From the Forearm's Longitudinal Axis (Experiment 1)

\begin{tabular}{ccccc}
\hline Splint + Forearm & $I_{1}$ & $I_{2}$ & $I_{3}$ & $e_{3}$ \\
\hline A & 945.30 & 905.98 & 54.12 & $6.3^{\circ} \mathrm{CCW}$ \\
S & 945.30 & 895.70 & 64.40 & $0^{\circ}$ \\
B & 945.30 & 905.98 & 54.12 & $6.3^{\circ} \mathrm{CW}$ \\
\hline
\end{tabular}

Note-CW, clockwise; CCW, counterclockwise. 
(a)

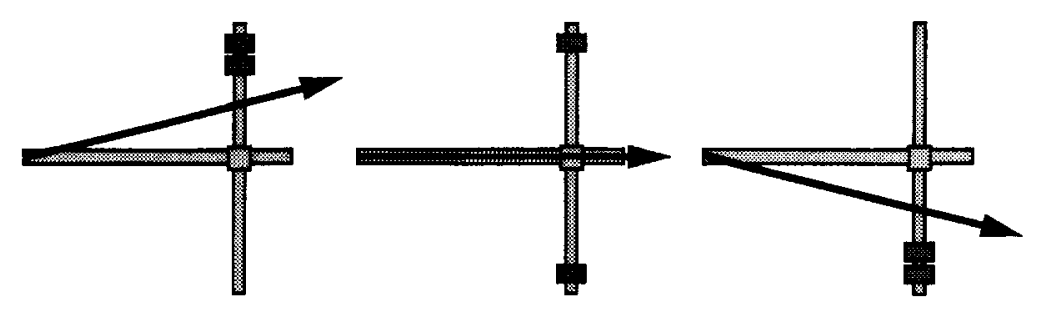

(b)

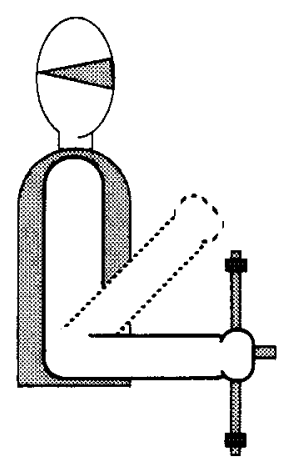

(c)

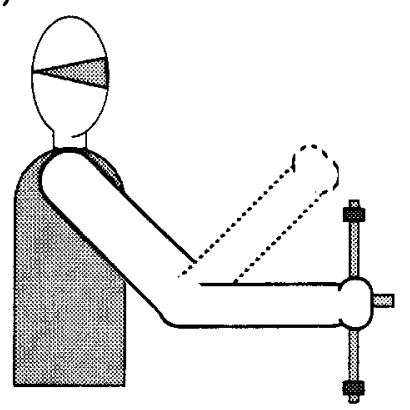

Figure 2. Experimental objects and conditions. (a) The three splint configurations with eigenvector rotation depictions of attached masses: above, symmetrical, and below, corresponding to counterclockwise rotation, no rotation, and clockwise rotation, respectively. The horizontal $0^{\circ}$ (solid lines) and incline $45^{\circ}$ (dotted lines) task orientations are shown in the elbow backward condition (b) and elbow forward condition (c).

45 min per subject. The subjects received no feedback about their performance during the course of the experiment.

There were 12 different conditions: 3 (splints) $\times 2$ (elbow positions) $\times 2$ (forearm orientations). As noted, both values of forearm orientation occurred on each trial. Accordingly, over the 12 trials per subject, each combination of splint and elbow position occurred twice, yielding two repetitions per condition. Each subject received Splints A, S, and B in a different randomized order. Elbow position was blocked, with the order of the blocks varied across the trials and across the participants.

Data collection and reduction. Kinematic data regarding the motions of the subject's arm were collected using a three-dimensional sonic digitizer (Science Accessories Corporation, Shelton, CT) and associated MASS kinematic analysis software (Engineering Solutions, Columbus, $\mathrm{OH}$ ). To collect motion data on the oscillating limbs, a high-frequency sound emitter $(3 \mathrm{~cm}$ long $\times .5 \mathrm{~cm}$ wide) was attached to the distal tip of each cross-like object, with another emitter at the elbow. The emitted sounds were detected by four microphones aligned in a plane perpendicular to the ground plane and parallel to the frontal plane of the subject. The sonic digitizer calculated the $x y z$ coordinates of an emitter in three-space, and thus the distal tip of an arm + splint configuration, by detecting the distances from each microphone to the emitter. An emitter's signal was sampled at $90 \mathrm{~Hz}$ for the duration of each trial, passed through an $A / D$ converter, and stored on the hard disk of a microcomputer. Each individual trial lasted $30 \mathrm{sec}$. Given that only final po- sitions were of interest, the data were down-sampled to $25 \mathrm{~Hz}$ before analysis.

The time series for each $30-\mathrm{sec}$ trial consisted of 750 sets of $x y z$ coordinates for the distal tip of each splint, one set every .04 sec. Each set of points was converted into an angle within the $y z$ plane. with $0^{\circ}$ corresponding to the forearm horizontal, and $+90^{\circ}$ corresponding to the forearm oriented vertically upward. The $y z$ planc was parallel to the midsagittal plane and passed through the point of rotation in the elbow. The angles for only the points in time when the subject was attempting to achieve a task-defined target orientation were averaged.

\section{Results and Discussion}

Tables 2 and 3 show the mean forearm orientations of each subject for the intended orientations of $0^{\circ}$ and $45^{\circ}$ as a function of splint. Table 2 summarizes the data for the forward elbow position, and Table 3 summarizes the data for the backward elbow position. Inspection of the tables reveals that subjects often approximated but rarely achieved exactly the required orientations. Further inspection suggests that although subjects may not have produced the required orientations perfectly, they were reliable in their productions: The separation between the two forearm orientations produced by an individual sub- 
Table 2

Produced Forearm Orientation (in Degrees) as a Function of Target Forearm Orientation (in Degrees) and Splint for the Subjects in Experiment 1 With the Elbow Forward

\begin{tabular}{crrrr}
\hline & & \multicolumn{3}{c}{ Splint } \\
\cline { 3 - 5 } Subject & Target Orientation & \multicolumn{1}{c}{ A } & \multicolumn{1}{c}{ S } & B \\
\hline 1 & 0 & 8.15 & 9.14 & 13.28 \\
& 45 & 34.07 & 36.93 & 41.17 \\
2 & 0 & 13.30 & 16.29 & 19.27 \\
& 45 & 38.93 & 41.01 & 43.26 \\
3 & 0 & 17.46 & 16.02 & 21.13 \\
& 45 & 36.12 & 32.37 & 39.66 \\
4 & 0 & 5.32 & 2.91 & 2.36 \\
& 45 & 31.32 & 33.01 & 30.46 \\
5 & 0 & 11.76 & 10.16 & 15.86 \\
& 45 & 40.19 & 45.01 & 50.64 \\
6 & 0 & 4.48 & 5.50 & 9.21 \\
& 45 & 27.39 & 26.12 & 29.49 \\
7 & 0 & 6.34 & 3.32 & 11.03 \\
& 45 & 34.01 & 33.35 & 38.90 \\
8 & 0 & 18.74 & 17.52 & 24.88 \\
& 45 & 42.19 & 38.41 & 44.55 \\
9 & 0 & 12.10 & 11.83 & 14.73 \\
& 45 & 41.13 & 44.10 & 45.43 \\
$M$ & 0 & 10.85 & 10.30 & 14.67 \\
& 45 & 36.15 & 36.70 & 40.40 \\
\hline
\end{tabular}

ject seems to have been of the same magnitude for each of the experimental conditions.

The major expectation was that the forearm would be oriented higher with $\left\{e_{k}\right\}$ rotated $6.3^{\circ} \mathrm{CW}$ from the arm's longitudinal axis (Splint B) than with $\left\{e_{k}\right\}$ rotated $6.3^{\circ}$ $\mathrm{CCW}$ from the arm's longitudinal axis (Splint A), with the $0^{\circ}$ deflection (Splint S) leading to an intermediate orientation. Overall, the mean forearm orientations (averaged over the intended orientations of $0^{\circ}$ and $45^{\circ}$ ) were $24.8^{\circ}$, $20.5^{\circ}$, and $20.1^{\circ}$, for Splint B, Splint S, and Splint A, respectively. A $2 \times 3 \times 2$ analysis of variance (ANOVA) with within-subjects factors of splint, elbow position, and forearm orientation revealed that the effect of splint was highly significant $[F(2,16)=21.2, p<.0001]$. A Tukey honestly significant difference (HSD) test determined that of the three levels of splint, Splint B differed from both Splint A and Splint $S(p<.001)$, but Splint A did not differ from Splint $S(p>.05)$. Also significant were forearm orientation $\left(9.5^{\circ}\right.$ when attempting $0^{\circ}$ and $34.1^{\circ}$ when attempting $\left.45^{\circ}\right)[F(1,8)=281.1, p<.0001]$ and elbow position $\left(18.7^{\circ}\right.$ for the backward position in which the upper arm was vertical vs. $24.8^{\circ}$ for the forward position in which the upper arm was $45^{\circ}$ to the vertical $)[F(1,8)=$ $19.4, p<.002]$. The two additional expectations were that the effect of $\left\{e_{k}\right\}$ 's rotation would be the same for both intended forearm orientations and for both elbow positions. In agreement, the ANOVA showed that splint did not interact with either forearm orientation or elbow position and that there was no three-way interaction (all $F$ s $<1$ ). The only significant interaction was that between elbow position and forearm orientation $[F(1,8)=$ $20.7, p<.002]$, indicating that subjects produced the two forearm orientations differentially as a function of elbow position.

The main effect of forearm orientation shows that subjects did indeed direct the forearm in two distinct directions according to instructions, at $9.5^{\circ}$ on the average when attempting a forearm orientation of $0^{\circ}$ and at $34.1^{\circ}$ on the average when attempting a forearm orientation of $45^{\circ}$. The main effect of splint and the absence of any interactions involving splint support the hypothesis that the position sense is affected by the eigenvectors of the forearm's inertia tensor (Pagano et al., 1996; Pagano \& Turvey, 1995). That is, the deflections of the forearm's inertia ellipsoid from the forearm's longitudinal spatial axis, the rotation of $\left\{e_{k}\right\}$ relative to $\left\{\sigma_{k}\right\}$, affected both of the intended forearm directions and did so in the same way independently of the angle made by the forearm with the upper arm. The independence of the influence of $\left\{e_{k}\right\}$ from elbow angle lends further support to the idea that absolute orientation-a direction in three-space - is of greater relevance to the position sense than relative orientation (see, e.g., Soechting, 1982; Soechting \& Ross, 1984; Worringham \& Stelmach, 1985; Worringham et al., 1987).

Although the predictions from the eigenvector hypothesis were satisfied qualitatively by the data, they were satisfied less well quantitatively. If the felt position of the forearm corresponded straightforwardly to the forearm's physical frame $\left\{e_{k}\right\}$, then Splint B should have been associated with a forearm orientation that was $12.6^{\circ}$ different from that associated with Splint $A$. The actual difference was less than $5^{\circ}$. Moreover, the expected $6.3^{\circ}$ difference between the orientations of Splint $S$ and Splint A was in actuality less than $1^{\circ}$. There is a possi-

Table 3

Produced Forearm Orientation (in Degrees) as a Function of Target Forearm Orientation (in Degrees) and Splint for the Subjects in Experiment 1 With the Elbow Backward

\begin{tabular}{crrrr}
\hline & & \multicolumn{3}{c}{ Splint } \\
\cline { 3 - 5 } Subject & Target Orientation & A & \multicolumn{1}{c}{ S } & B \\
\hline 1 & 0 & 7.13 & 11.25 & 16.22 \\
& 45 & 34.05 & 36.08 & 40.54 \\
2 & 0 & 3.93 & 6.51 & 12.39 \\
& 45 & 25.45 & 31.27 & 36.24 \\
3 & 0 & 8.30 & 8.19 & 11.42 \\
& 45 & 27.65 & 25.40 & 25.99 \\
4 & 0 & -7.23 & -2.56 & -.60 \\
& 45 & 20.09 & 19.51 & 21.33 \\
5 & 0 & 7.93 & 14.85 & 15.11 \\
& 45 & 38.98 & 41.84 & 47.16 \\
6 & 0 & 1.43 & -2.68 & 7.29 \\
& 45 & 24.77 & 19.47 & 26.27 \\
7 & 0 & -3.04 & -6.36 & -1.36 \\
& 45 & 17.25 & 16.34 & 31.32 \\
8 & 0 & 15.06 & 13.54 & 18.85 \\
& 45 & 31.82 & 33.08 & 37.02 \\
9 & 0 & 10.09 & 11.96 & 11.29 \\
& 45 & 36.13 & 36.27 & 39.22 \\
$M$ & 0 & 4.84 & 6.08 & 10.07 \\
& 45 & 28.46 & 28.81 & 33.90 \\
\hline
\end{tabular}


Table 4

Major $\left(I_{1}\right)$, Intermediate $\left(I_{2}\right)$, and Minor $\left(I_{3}\right)$ Eigenvalues $\left(\mathrm{g} \cdot \mathrm{cm}^{2} / 1,000\right)$ for the Weighted Three Splints Plus Forearm Relative to the Rotation Point in the Elbow Together With the Deflections of the Forearm's Eigenvector $e_{3}$ From the Forearm's Longitudinal Axis (Experiment 2)

\begin{tabular}{ccccc}
\hline Splint + Forearm & $I_{1}$ & $I_{2}$ & $I_{3}$ & $e_{3}$ \\
\hline S & 945.30 & 895.70 & 64.40 & $0^{\circ}$ \\
B & 945.30 & 905.98 & 54.12 & $6.3^{\circ} \mathrm{CW}$ \\
B $^{*}$ & $1,098.5$ & 954.75 & 163.55 & $15.5^{\circ} \mathrm{CW}$ \\
\hline
\end{tabular}

Note-CW, clockwise.

bility that the limited quantitative fit may have been due, in part, to aspects of the experimental arrangement. The interaction of forearm orientation with elbow position arose from a greater influence of elbow position on the intended $45^{\circ}$ forearm orientation (forward, $37.7^{\circ}$, vs. backward, $30.4^{\circ}$ ) than on the intended $0^{\circ}$ forearm orientation (forward, $11.9^{\circ}$, vs. backward, $7^{\circ}$ ). Specifically, for some subjects, the chosen $45^{\circ}$ forearm orientation in the elbow back position was at the limit of the range of possible movement. Accordingly, the $45^{\circ}$ forearm orientation was less closely approximated for the elbow back than for the elbow forward. Another experimental artifact may have contributed to the insignificant difference between Splint A and Splint S. Casual inspection of subject's behavior suggested that they had to provide a counterrotational torque in order to stabilize turning of the forearm by Splint A. This additional effort, standing in sharp contrast to what was required to preserve Splint $S$ and Splint B in a sagittoparallel plane, may have interfered with the effect of rotating $\left\{e_{k}\right\}$.

\section{EXPERIMENT 2}

Experiment 2 was designed to corroborate the findings of Experiment 1, with particular reference to the possibly artifactual influence of elbow position. Experiment 2 was identical in design to Experiment 1 with two major exceptions. Because of the apparent instability of the forearm posture when the splint was weighted above the longitudinal axis, only splints weighted below or weighted equally above and below were used. In addition, in an attempt to circumvent the apparent difficulty in producing the $45^{\circ}$ forearm orientation in the backward position of the elbow, each subject was made aware of the possibility that the biceps could restrict the range of motion if the arm was positioned steeper than $45^{\circ}$.

Corresponding to the inertial eigenvector hypothesis and the results of Experiment 1, the expected outcomes were that (1) the forearm would be oriented higher, the greater the $\mathrm{CW}$ rotation of $\left\{e_{k}\right\}$ relative to $\left\{\sigma_{k}\right\} ;(2)$ the degree to which forearm orientation was affected by $\left\{e_{k}\right\}$ 's direction would be the same for both intended forearm orientations, and (3) the degree to which forearm orientation was affected by $\left\{e_{k}\right\}$ 's direction would be the same for both elbow positions. On the assumption that the experimental modifications addressed the "artifact" issues of Experiment 1, a further expected outcome was that (4) elbow position (and, therefore, joint angle) would not be influential either as a main effect or interactively.

\section{Method}

Subjects. Ten students ( 6 men and 4 women) associated with the University of Connecticut participated on a volunteer basis. None had participated in Experiment 1. All were right-handed. No subject had any foreknowledge of the specific hypothesis in question or of the experimental conditions used.

Materials. Splint B and Splint S from Experiment 1 were used together with two additional splints. One additional splint, referred to as Splint $\mathrm{S}^{*}$, was identical in dimensions to Splint $\mathrm{S}$ but without the added metal rings. Splint $S^{*}$ was introduced to evaluate whether the deviations in Experiment 1 of the forearm with Splint $S$ from the intended orientations were due to the weight of Splint $S$. The other additional splint, referred to as Splint $\mathrm{B}^{*}$, was distinguished by a $30-\mathrm{cm}$ branch extending below the center of the brace with a $200-\mathrm{g}$ metal ring attached. The eigenvalues and $e_{3}$ deflections of the four forearm + splint conditions are summarized in Table 4 .

Procedure, data collection, and data analysis. These were identical to those of Experiment 1.

\section{Results and Discussion}

Tables 5 and 6 show the mean forearm orientations of each subject for the intended orientations of $0^{\circ}$ and $45^{\circ}$ as a function of splint. Table 5 summarizes the data for the forward elbow position, and Table 6 summarizes the data for the backward elbow position. As for the corresponding tables of Experiment 1, inspection of Tables 5 and 6 reveals that subjects often approximated but rarely achieved the required orientations exactly. In further agreement with Experiment 1, inspection of Tables 5 and 6 suggests that although subjects may not have produced the re-

Table 5

Produced Forearm Orientation (in Degrees) as a Function of Target Forearm Orientation (in Degrees) and Splint for the Subjects in Experiment 2 With the Elbow Forward

\begin{tabular}{|c|c|c|c|c|c|}
\hline \multirow[b]{2}{*}{ Subject } & \multirow[b]{2}{*}{ Target Orientation } & \multicolumn{4}{|c|}{ Splint } \\
\hline & & $\mathrm{B}^{*}$ & B & $\mathrm{S}$ & $\mathrm{S}^{*}$ \\
\hline \multirow[t]{2}{*}{1} & 0 & 0.91 & -4.49 & -7.06 & -8.08 \\
\hline & 45 & 48.16 & 39.99 & 28.65 & 32.63 \\
\hline \multirow[t]{2}{*}{2} & 0 & 12.11 & 9.71 & 8.81 & 7.23 \\
\hline & 45 & 49.43 & 50.12 & 42.15 & 44.66 \\
\hline \multirow[t]{2}{*}{3} & 0 & -1.11 & -3.86 & -8.70 & -2.88 \\
\hline & 45 & 29.34 & 26.66 & 22.86 & 34.82 \\
\hline \multirow[t]{2}{*}{4} & 0 & 15.89 & 12.02 & 6.74 & 8.42 \\
\hline & 45 & 49.55 & 45.47 & 39.00 & 35.08 \\
\hline \multirow[t]{2}{*}{5} & 0 & 22.13 & 19.23 & 17.29 & 16.57 \\
\hline & 45 & 45.28 & 45.66 & 41.35 & 27.58 \\
\hline \multirow[t]{2}{*}{6} & 0 & 20.68 & 15.51 & 9.57 & 10.44 \\
\hline & 45 & 45.76 & 36.57 & 23.88 & 31.34 \\
\hline \multirow[t]{2}{*}{7} & 0 & 18.51 & 19.17 & 13.99 & 17.65 \\
\hline & 45 & 42.00 & 34.08 & 40.72 & $40.0 \mathrm{l}$ \\
\hline \multirow[t]{2}{*}{8} & 0 & -1.37 & -4.98 & -0.88 & -5.81 \\
\hline & 45 & 28.24 & 31.23 & 29.07 & 26.53 \\
\hline \multirow[t]{2}{*}{9} & 0 & 16.50 & 11.15 & 7.51 & 7.22 \\
\hline & 45 & 32.36 & 34.37 & 24.83 & 30.87 \\
\hline \multirow[t]{2}{*}{10} & 0 & 2.42 & 2.36 & 2.74 & -0.44 \\
\hline & 45 & 13.46 & 20.54 & 17.13 & 21.92 \\
\hline \multirow[t]{2}{*}{$M$} & 0 & 10.67 & 7.58 & 5.00 & 5.03 \\
\hline & 45 & 38.36 & 36.47 & 30.96 & 32.54 \\
\hline
\end{tabular}


Table 6

Produced Forearm Orientation (in Degrees) as a Function of Target Forearm Orientation (in Degrees) and Splint for the Subjects in Experiment 2 With the Elbow Backward

\begin{tabular}{crrrrr}
\hline & & \multicolumn{4}{c}{ Splint } \\
\cline { 3 - 6 } Subject & Target Orientation & \multicolumn{1}{c}{$\mathrm{B}^{*}$} & \multicolumn{1}{c}{$\mathrm{B}$} & \multicolumn{1}{c}{$\mathrm{S}$} & \multicolumn{1}{c}{$\mathrm{S}^{*}$} \\
\hline 1 & 0 & 12.70 & 18.19 & 10.86 & 10.52 \\
& 45 & 40.13 & 38.84 & 33.71 & 35.28 \\
2 & 0 & 7.36 & 4.44 & 1.20 & 3.28 \\
& 45 & 33.30 & 31.33 & 24.52 & 26.88 \\
3 & 0 & -2.84 & 1.29 & -.463 & -1.79 \\
& 45 & 28.36 & 28.90 & 25.89 & 26.93 \\
4 & 0 & 15.91 & 17.56 & 6.62 & 10.11 \\
& 45 & 43.71 & 43.37 & 32.00 & 33.46 \\
5 & 0 & 23.17 & 17.31 & 9.89 & 12.83 \\
& 45 & 39.65 & 37.20 & 28.36 & 33.46 \\
6 & 0 & 12.69 & 9.95 & 6.92 & 8.67 \\
& 45 & 32.89 & 28.66 & 27.52 & 27.03 \\
7 & 0 & 14.02 & 12.63 & 8.75 & 10.77 \\
& 45 & 31.70 & 34.89 & 30.91 & 33.85 \\
8 & 0 & 1.74 & -6.96 & -5.05 & -7.50 \\
& 45 & 26.39 & 24.49 & 20.88 & 23.17 \\
9 & 0 & 14.28 & 9.00 & 7.44 & 7.47 \\
& 45 & 44.27 & 34.83 & 31.06 & 33.83 \\
10 & 0 & 0.29 & 0.09 & -3.18 & 0.22 \\
& 45 & 12.67 & 13.40 & 12.71 & 14.21 \\
$M$ & 0 & 9.93 & 8.35 & 4.3 & 5.46 \\
& 45 & 33.31 & 31.59 & 26.76 & 29.70 \\
\hline & & & & &
\end{tabular}

quired orientations perfectly, they were reliable in their productions: The separation between the two forearm orientations produced by an individual subject seems to have been of the same magnitude for each of the experimental conditions.

The major prediction was that the forearm would be oriented higher, the greater the $\mathrm{CW}$ rotation of $\left\{e_{k}\right\}$ relative to the arm's longitudinal axis. For Splints $\mathrm{B}^{*}, \mathrm{~B}, \mathrm{~S}$, and $\mathrm{S}^{*}$, corresponding to $\left\{e_{k}\right\}$ rotations of $16.3^{\circ} \mathrm{CW}, 6.3^{\circ}$ $\mathrm{CW}, 0^{\circ}$, and $0^{\circ}$, respectively, the mean forearm orientations (averaged over intended $0^{\circ}$ and $45^{\circ}$ ) were $23.1^{\circ}, 20.9^{\circ}$, $16.8^{\circ}$, and $18^{\circ}$, respectively. A $2 \times 4 \times 2$ ANOVA with within-subjects factors of splint, elbow position, and forearm orientation revealed that the effect of splint was highly significant $[F(3,27)=17.96, p<.0001]$. A Tukey HSD test revealed that Splint $B^{*}$ differed reliably from Splint B $(p<.05)$, that both Splints B ${ }^{*}$ and B differed reliably from both Splints $S$ and $S^{*}(p<.001)$, and that there was no significant difference between Splint $S$ and Splint $\mathrm{S}^{*}(p>.05)$. The equivalence of Splint $\mathrm{S}$ (added metal rings) and Splint $S^{*}$ (no added metal rings) indicates that weight of the splints was not a factor determining forearm orientation. As in Experiment 1, forearm orientation $\left(7^{\circ}\right.$ when the target orientation was $0^{\circ}$ and $32.4^{\circ}$ when the target orientation was $45^{\circ}$ ) was highly significant $[F(1,9)=171.9, p<.0001]$. Contrary to Experiment 1 , and in agreement with what was expected from the experimental modifications, elbow position $\left(18.6^{\circ}\right.$ for the backward position in which the upper arm was vertical vs. $20.8^{\circ}$ for the forward position in which the upper arm was $45^{\circ}$ to the vertical) was not significant $[F(1,9)<1]$, nor was the interaction of elbow position with forearm orientation $[F(3,27)=3.92, p>.05]$. The two additional expectations were that the effect of $\left\{e_{k}\right\}$ 's direction would be the same for both intended forearm orientations and for both elbow positions. In agreement, the ANOVA found that splint did not interact with either forearm orientation or elbow position and that there was no three-way interaction (all $F$ s $<1$ ).

In summary, the results of Experiment 2 confirmed the basic predictions of the eigenvector hypothesis. Positioning the forearm in two orientations $-0^{\circ}$ and $45^{\circ}$ relative to the ground plane - was systematically influenced by the direction of the forearm's eigenvectors. The size of this influence was the same for both intended orientations and for both forward and backward positions of the elbow. The independence from orientation suggests that gravitational torque was not a factor. A near horizontal forearm posture would have been associated with larger gravitational torques than a near $45^{\circ}$ forearm posture. The observation that produced forearm orientation was the same for the two symmetric splints, Splint $S$ and Splint $\mathrm{S}^{*}$, despite the 200 -g difference between them, also runs counter to the notion that the position sense uses gravitational torque.

As in Experiment 1, the shift in limb position due to the eigenvector manipulations was consistently less than predicted. The changes introduced in Experiment 2 did not improve the quantitative fit. In Pagano et al. (1996), the shift in subjects' perceptions corresponded to approximately one third of the $\left\{e_{k}\right\}$ rotation. They noted that this was considerably less than the shift observed in related experiments (Pagano \& Turvey, 1995, Experiments 1 and 2) in which subjects executed exploratory movements with limb plus splint before producing the targeted limb position. A possible implication is that in the limb matching task used by Pagano et al. (1996), and in the single limb environmental positioning task of the present experiments, subjects do not have the opportunity on any single trial to become fully attuned to the new rotational inertia characteristics of their limbs.

\section{GENERAL DISCUSSION}

The present results provide further evidence that the positioning of limbs and the perception of their positions can be reliably biased by manipulations of the limbs' inertia tensor. The broad implication of such results is that the inertial properties of the limbs have proprioceptive consequences, an implication in concert with the findings of Ghez, Gordon, and colleagues on the guidance of limb movements (Ghez, Gordon, Ghilardi, Christakos, \& Cooper, 1990; Ghez, Gordon, Ghilardi, \& Sainburg, 1995; Gordon, Ghilardi, \& Ghez, 1994) and with the "mobility tensor" perspective on multijoint posture and movement control (Hogan. 1985; Mussa-Ivaldi, Hogan, \& Bizzi, 1985). A particularly significant feature of the present results in respect to the eigenvectors hypothesis is that the effect of $\left\{e_{k}\right\}$ was independent of other manipulations of potential relevance to proprioception. To reiterate, rotat- 
ing $\left\{\boldsymbol{e}_{k}\right\}$ relative to $\left\{\sigma_{k}\right\}$ affected perception of forearm position independently of the intended forearm direction, the angle of the forearm relative to the upper arm, and the weight of the forearm.

It was suggested above (in the Results and Discussion section of Experiment 2) that the less than expected degree of perceptual shift was due to limited experience with the new eigenvectors. A different interpretation is that the afferent consequences of manipulating the forearm's inertia ellipsoid are not absolute but rather are relative to the motor organization of the limb that fixes a particular posture. The latter is the key idea in Feldman and Latash's (1982a, 1982b) approach to the position sense. In terms of the $\lambda$ hypothesis on the control of discrete arm movements (Feldman, 1986; Feldman \& Levin, 1995 ), one posture is transformed into another by an $R$ command. The $\lambda$ values (threshold values of tonic stretch reflexes) of the elbow flexors and extensors that define the initial posture are unidirectionally shifted by an $R$ command, with a consequent change in the limb configuration to that posture at which active muscular and imposed torques balance. The sensed position in terms of joint angle $\theta$ can be obtained from the relation

$$
\theta=R+f_{c}^{-1}\left(M-M_{R}\right),
$$

where the subscript $C$ refers to the coactivation command that establishes the compliance of the joint around its $R$ value, $M$ refers to the total muscular torque at the joint, and $M_{R}$ refers to the passive muscular torque for $\theta=R$. What the preceding equation shows is that the transformed afferent state reflecting active muscle torque due to limb position and loading is measured relative to $R$. From a perspective such as that proposed by Feldman and Latash (1982a, 1982b; see also Feldman \& Levin, 1995), it would not be surprising to find that the shift in perceived limb direction does not conform to the actual shift in the direction of the limb's inertia ellipsoid. Indeed, the form of the equation above suggests that if the CNS can determine the point $\left(M_{R}, R\right)$, then perceived joint angle will be a logarithmic function of the muscle torque $M$ (Feldman \& Latash, 1982a).

The effect of rotating $\left\{e_{k}\right\}$ relative to $\left\{\sigma_{k}\right\}$ paralleis a number of so-called illusory effects of limb position that occur as a result of tendon vibration of a secured arm and as a result of a briefly sustained prior limb position (see Jones, 1988, for a review). A vibrated limb is felt to be at a position other than its actual spatial position. In Goodwin, McCloskey, and Mathews (1972) original experiments, mean error in matching one limb with another, when one of them was vibrated, ranged between $5.5^{\circ}$ and $8^{\circ}$. In Pagano et al.'s (1996) experiments, mean error in matching one limb with another, when $\left\{e_{k}\right\}$ of one limb was rotated relative to $\left\{e_{k}\right\}$ of the other limb, ranged between approximately $1^{\circ}$ and $6.5^{\circ}$, depending on the magnitude and direction of the rotation. The modified position sense due to vibration has been attributed to the disruption of the afferent-efferent relationship in normal neural activity (Burgess et al., 1982; Feldman \& Latash, 1982a, 1982b; Gilhodes et al., 1992). The similarly modified position sense due to manipulation of $\left\{e_{k}\right\}$ is not readily attributable to a disruption of normal activity but may, nonetheless, be understandable in terms of afferentefferent relations. The fact that illusions of limb posture can occur that require extrapolation beyond previous experience (Craske, 1977; Lackner \& Taublieb, 1984), and that adjacent limb segments can feel nonadjacent or occupying the same position (Lackner \& DiZio, 1984; Lackner \& Taublieb, 1984), highlights the on-line nature of the sense of position and dilutes the arguments for either a memory-based or an anatomically based internal model of the body. The latter facts also question a sense of joint angle. The induced changes in the apparent lengths of muscles are not canceled by signals from the joints indicating that there has been no corresponding change of joint angles.

The effect in the present experiments of rotating $\left\{e_{k}\right\}$ relative to $\left\{\sigma_{k}\right\}$ can be considered, in light of the illusion research, as a shift of body image. ${ }^{1}$ It implies that the haptic perceptual system is sensitive to the patterning of afferent activity induced by $\left\{e_{k}\right\}$ in such a way that when the physical frame $\left\{e_{k}\right\}$ is made noncoincident with the spatial frame $\left\{\sigma_{k}\right\}$, the impression or "image" of the limb's position goes with $\left\{e_{k}\right\}$. This consequence of rotating $\left\{e_{k}\right\}$ relative to $\left\{\sigma_{k}\right\}$ complements the vibration-induced changes in limb position. Stretching a muscle is known to enhance the responses of sensory endings to vibration (Burke, Hagbarth, \& Löfstedt, 1978). The lesson, perhaps, of the present experiments is that a shift in body image may be dependent on changes in muscular-tendinous states that are defined in terms more abstract than the simple notion of muscle length. It has been argued that the relevant quantification of muscular-tendinous states is through a deformation tensor defined globally over the muscles acting synergistically at a joint (e.g., Pagano, Fitzpatrick, \& Turvey, 1993; Solomon, 1988; Turvey \& Carello, 1995). Consonant with the latter argument are efforts to understand how an agonist and antagonist might code joint posture through their collective action (e.g., Burgess et al., 1982; Gilhodes et al., 1992) and how the shape of a hydrostatic organ without a joint, such as the tongue or an elephant's trunk or a tentacle, might play a role in perceiving the organ's position (Grover \& Craske, 1991; Smith \& Kier, 1989).

With respect to other illusions reviewed by Jones (1988), aspects of the present results conform to the so-called phenomenon of postural persistence investigated by Hoff and Schilder (1925), Selling (1930), and Jackson (1954). Specifically, in Experiments 1 and 2 the forearm with Splints $\mathrm{S}$ and $\mathrm{S}^{*}$ was judged horizontal when it was above the horizon. In addition, in the two experiments, the forearm positions in all asymmetrical splint conditions were upwardly rotated relative to the direction expected from $\left\{e_{k}\right\}$. As Jackson noted, there is a "constant upwards effect" evident in the efforts of people to orient an upper limb or limb segment at a prescribed inclination. 
Finally, the present results should be considered in the light of other manifestations of the position sense's lability, specifically its tendency to drift over time in the absence of vision (e.g., Gross, Webb, \& Melzak, 1974; Wann \& Ibrahim, 1992) and its sensitivity to prior positions (e.g., Craske \& Cranshaw, 1974; Howard \& Anstis, 1974; Jackson, 1954). These features may demand a measure of limb position that includes explicitly the effects of noise-such as the target resolution metric based in information theory proposed by Clark and colleagues (Clark, Larwood, Davis, \& Deffenbacher, 1995). Drift and the influences of immediately previous postures may have been at work in Experiments 1 and 2. The predictions, however, were primarily in respect to the relative orderings of limb positions due to the different rotations of $\left\{e_{k}\right\}$ relative to $\left\{\sigma_{k}\right\}$ rather than the precision of those limb positions.

In summary, the results of the present experiments provide further evidence for the hypothesis that perceived limb position is constrained by the limb's physical frame $\left\{e_{k}\right\}$ rather than its spatial frame $\left\{\sigma_{k}\right\}$. This hypothesis has now received support in three different perceptionaction tasks: Pointing about the shoulder to visual targets with an occluded arm (Pagano \& Turvey, 1995); matching one occluded forearm with the other (Pagano et al., 1996); and orienting an occluded forearm in particular spatial directions at different joint angles (the present research). For the understanding of movement control and coordination, these experimental outcomes suggest that issues such as "trajectory planning" should be addressed in terms of the relative directions of the segmental inertia ellipsoids rather than joint angles.

\section{REFERENCES}

Barac-Cikoja, D., \& Turvey, M. T. (1993). Perceiving aperture size by striking. Journal of Experimental Psychology: General, 122, 347-370.

Bermúdez, J. L., Marcel, A., \& Eilan, N. (1995). The body and the self. Cambridge, MA: MIT Press.

BERNSTEIN, N. A. (1996). Levels of construction of movement. In M. L. Latash \& M. T. Turvey (Eds.), Dexterity and its development (pp. 115-170). Mahwah, NJ: Erlbaum.

Burgess, P. R., Clark, F. J., Simon, J., \& Wel, J. Y. (1982). Signaling of kinesthetic information by peripheral sensory receptors. Annual Review of Neuroscience, 5, 171-187.

Burke, D., Hagbarth, K. E., \& Löfstedt, L. ( 1978). Muscle spindle activity in man during shortening and lengthening contractions. Journal of Physiology, 277, 131-142.

Chandler, R. F., Clauser, C. E., McContville, J. P., Reynolds, H. M., \& YounG, J. W. (1975). Investigation of inertial properties of the human body: Final report, Apr. 1, 1972-Dec. 1974 (AMRL-TR74-137). Dayton, OH: Wright-Patterson Air Force Base, Aerospace Medical Research Laboratories.

Clark, F. J., Larwood, K. J., Davis, M. E., \& Deffenbacher, K. A. (1995). A metric for assessing acuity in positioning joints and limbs. Experimental Brain Research, 107, 73-79.

Clauser, C. E., McContville, J. P., \& Young, J. W. (1969). Weight. volume and center of mass of segments of the human body (AMRLTR-69-70). Dayton, OH: Wright-Patterson Air Force Base, Aerospace Medical Research Laboratories.

COLE, I. (1995). Pride and a daily marahon. Cambridge, MA: MIT Press.

COLE. J.. \& PAIL.LARD, J. (1995). Living without touch and peripheral information about body position and movement: Studies with deaffer- ented subjects. In J. L. Bermúdez, A. Marcel, \& N. Eilan (Eds.), The body and the self (pp. 245-266). Cambridge, MA: MIT Press.

CRASKE, B. (1977). Perception of impossible limb positions induced by tendon vibration. Science, 196, 71-73.

Craske, B., \& Cranshaw, M. (1974). Differential errors of kinesthesis produced by previous limb positions. Journal of Motor Behavior, 6, 273-278.

FELDMAN, A. G. (1986). Once more on the equilibrium point hypothesis (1-model) for motor control. Journal of Motor Behavior, 18, 17-54.

Feldman, A. G., \& Latash, M. L. (1982a). Afferent and efferent components of joint position sense: Interpretation of kinaesthetic illusions. Biological Cybernetics, 42, 205-214.

FELDMAN, A. G., \& LATASH, M. L. (1982b). Interaction of afferent and efferent signals underlying joint position sense: Empirical and theoretical approaches. Journal of Motor Behavior, 14, 174-193.

Feldman, A. G., \& LEVIN, M. L. (1995). The origin and use of positional frames of reference in motor control. Behavioral \& Brain Sciences, 18, 723-806.

Ghez, C., Gordon, J., Ghilardi, M. F., Christakos, C. N., \& Cooper, S. E. (1990). Roles of proprioceptive input in the programming of arm trajectories. Cold Spring Harbor Symposia on Quantitative Biology, LV, 837-847.

Ghez, C., Gordon, J., Ghilardi, M. F., \& Sainburg, R. (1995). Contributions of vision and proprioception to accuracy in limb movements. In M. S. Gazzaniga (Ed.), The cognitive neurosciences (pp. 549-564). Cambridge, MA: MIT Press.

GiBson, J. J. (1966). The senses considered as perceptual systems. Boston: Houghton Mifflin.

Gilhodes, J. C., Colton, Y., Roll, J. P., \& ANs, B. (1992). Propriomuscular coding of kinaesthetic sensation. Biological Cybernetics, 68, 509-517.

Goodwin, G. M., McCloskey, D. I., \& Mathews, P. B. C. (1972). The contribution of muscle afferents to kinesthesia shown by vibration induced illusions of movement and by the effects of paralyzing joint afferents. Brain, 96, 705-748.

Gordon, J., Ghilardi, M., \& Ghez, C. (1994). Accuracy of planar reaching movements: II. Systematic extent errors resulting from inertial anisotropy. Experimental Brain Research, 99, 112-130.

Gross, Y., WebB, R., \& MelzaK, R. (1974). Central and peripheral contributions to localization of body parts: Evidence for a central body schema. Experimental Neurology, 44, 346-362.

Grover, C., \& CraSke, B. (1991). The effect of loading on position sense in the tongue. Perception \& Psychophysics, 50, 7-14.

HOFF, H., \& SCHILDER, P. (1925). Über Lagebeharrung [On postural persistence]. Monatsschrift für Psychiatrie und Neurologie, 58, 257-264.

HoGaN, N. (1985). The mechanics of multi-joint posture and movement control. Biological Cybernetics, 52, 315-331.

HowaRD, I. P., \& ANSTIS, T. (1974). Muscular and joint-receptor components in postural persistence. Journal of Experimental Psychologv. $103,167-170$.

JACKSON, C. V. (1954). The influence of previous movement and posture on subsequent posture. Quarterly Journal of Experimental PSychology, 6, 72-78.

JoNEs, L. A. (1988). Motor illusions: What do they reveal about proprioception? Psychological Bulletin, 103, 72-86.

LACKNER, J. R., \& DiZIO, P. (1984). Some efferent and somatosensory influences on body orientation and oculomotor control. In L. Spillmann \& B. R. Wooten (Eds.), Sensory experience, adaptation, and perception (pp. 281-301). Hillsdale, NJ: Erlbaum.

LaCkner, J. R., \& Taublieb, A. B. (1984). Influence of vision on vibration-induced illusions of limb movement. Experimental Nellrology, 85, 97-106.

LoOMis, J. M., \& Lederman, S. J. (1986). Tactile perception. In K. R. Boff, L. Kaufman, \& J. P. Thomas (Eds.), Handbook of perception and human performance (pp. 31.01-31.41). New York: Wiley.

Mussa-Ivaldi, F. A.. Hogan, N., \& Bizzi, E. (1985). Neural, mechani$\mathrm{Cal}$, and geometric factors subserving arm posture. Journal of Newoscience, 5, 2732-2743.

Pagano, C. C., Fitzpatrick, P., \& Turvey, M. T. (1993). Tensorial basis to the constancy of perceived object extent over variations of dynamic touch Perception \& Psuchophysics, 54, 43-54 
Pagano, C. C., Garrett, S., \& Turvey, M. T. (1996). Is limb proprioception a function of the limbs' eigenvectors? Ecological Psychology, 8, 43-69.

Pagano, C. C., \& Turvey, M. T. (1995). The inertia tensor as a basis for the perception of limb orientation. Journal of Experimental Psychology: Human Perception \& Performance, 21, 1070-1087.

REYNOLDS, H. M. (1978). The inertial properties of the body and its segments (NASA Reference Publication 1024, pp. IV-1 to IV-760. Washington, DC: Nasa Scientific and Technical Information Office.

SELLING, L. S. (1930). An experimental investigation of the phenomenon of postural persistence. Archives of Psychology, 18 (Whole No. 118).

SMITH, K. K., \& KIER, W. S. (1989). Trunks, tongues, and tentacles: Moving with skeletons of muscle. American Scientist, 77, 29-35.

SoECHTING, J. F. (1982). Does position sense at the elbow reflect a sense of elbow joint angle or one of limb orientation? Brain Research, 248 , 392-395.

Soechting, J. F., \& Ross, B. (1984). Psychophysical determination of coordinate representation of human arm orientation. Neuroscience, $13,595-604$.

Solomon, H. Y. (1988). Movement-produced invariants in haptic explorations: An example of self-organizing, information-driven, intentional system. Human Movement Science, 7, 201-223.

Turvey, M. T. (1996). Dynamic touch. American Psychologist, 51, $1134-1152$
Turvey, M. T., \& Carello, C. (1995). Dynamic touch. In W. E. S. Rogers (Ed.), Handbook of perception and cognition: V. Perception of space and motion (pp. 401-490). San Diego, CA: Academic Press. voN HoLST, E. (1954). Relation between the central nervous system and the peripheral organs. British Journal of Animal Behavior, 2, 89-94.

WanN, J. P., \& IBrahim, S. F. (1992). Does limb proprioception drift? Experimental Brain Research, 91, 162-166.

Worringham, C. J., \& Stelmach, G. E. (1985). The contribution of gravitational torques to limb position sense. Experimental Brain Research, 61, 38-42.

Worringham, C. J., Stelmach, G. E., \& Martin, Z. E. (1987). Limb segment inclination sense in proprioception. Experimental Brain Research, 66, 653-658.

\section{NOTE}

1. There is much confusion surrounding the usages of such terms as body image and body schema as highlighted in many of the papers in Bermúdez, Marcel, and Eilan's (1995) The body and the self.

(Manuscript received April 14, 1997; revision accepted for publication October $27,1997$. 